\title{
Generation of Porcine Induced Neural Stem Cells Using the Sendai Virus
}

\author{
Warunya Chakritbudsabong ${ }^{1,2}$, Ladawan Sariya ${ }^{3}$, Phakhin Jantahiran ${ }^{1,2}$, \\ Nattarun Chaisilp ${ }^{3}$, Somjit Chaiwattanarungruengpaisan ${ }^{3}$, Ruttachuk Rungsiwiwut ${ }^{4}$, \\ Joao N. Ferreira ${ }^{5,6}$ and Sasitorn Rungarunlert ${ }^{1,2 *}$
}

${ }^{1}$ Laboratory of Cellular Biomedicine and Veterinary Medicine, Faculty of Veterinary Science, Mahidol University, Nakhon Pathom, Thailand, ${ }^{2}$ Department of Preclinic and Applied Animal Science, Faculty of Veterinary Science, Mahidol University, Nakhon Pathom, Thailand, ${ }^{3}$ The Monitoring and Surveillance Center for Zoonotic Disease in Wildlife and Exotic Animals (MoZWE), Faculty of Veterinary Science, Mahidol University, Nakhon Pathom, Thailand, ${ }^{4}$ Department of Anatomy, Faculty of Medicine, Srinakharinwirot University, Bangkok, Thailand, ${ }^{5}$ Avatar Biotechnologies for Oral Health and Healthy Longevity Research Unit, Faculty of Dentistry, Chulalongkorn University, Bangkok, Thailand, ${ }^{6}$ Faculty of Dentistry, National University of Singapore, Singapore, Singapore

OPEN ACCESS

Edited by:

Fabiana Fernandes Bressan, University of São Paulo, Brazil

Reviewed by:

Wilfried A. Kues,

Friedrich Loeffler Institute

(FLI), Germany

Werner Giehl Glanzner, McGill University, Canada

*Correspondence:

Sasitorn Rungarunlert sasitorn.run@mahidol.edu

Specialty section:

This article was submitted to Veterinary Regenerative Medicine,

a section of the journal

Frontiers in Veterinary Science

Received: 01 November 2021

Accepted: 13 December 2021

Published: 12 January 2022

Citation:

Chakritbudsabong W, Sariya L, Jantahiran P, Chaisilp N, Chaiwattanarungruengpaisan $S$,

Rungsiwiwut R, Ferreira JN and Rungarunlert S (2022) Generation of Porcine Induced Neural Stem Cells

Using the Sendai Virus.

Front. Vet. Sci. 8:806785

doi: 10.3389/fvets.2021.806785
The reprogramming of cells into induced neural stem cells (iNSCs), which are faster and safer to generate than induced pluripotent stem cells, holds tremendous promise for fundamental and frontier research, as well as personalized cell-based therapies for neurological diseases. However, reprogramming cells with viral vectors increases the risk of tumor development due to vector and transgene integration in the host cell genome. To circumvent this issue, the Sendai virus (SeV) provides an alternative integration-free reprogramming method that removes the danger of genetic alterations and enhances the prospects of iNSCs from bench to bedside. Since pigs are among the most successful large animal models in biomedical research, porcine iNSCs (piNSCs) may serve as a disease model for both veterinary and human medicine. Here, we report the successful generation of piNSC lines from pig fibroblasts by employing the $\mathrm{SeV}$. These piNSCs can be expanded for up to 40 passages in a monolayer culture and produce neurospheres in a suspension culture. These piNSCs express high levels of NSC markers (PAX6, SOX2, NESTIN, and VIMENTIN) and proliferation markers (KI67) using quantitative immunostaining and western blot analysis. Furthermore, piNSCs are multipotent, as they are capable of producing neurons and glia, as demonstrated by their expressions of TUJ1, MAP2, TH, MBP, and GFAP proteins. During the reprogramming of piNSCs with the $\mathrm{SeV}$, no induced pluripotent stem cells developed, and the established piNSCs did not express OCT4, NANOG, and SSEA1. Hence, the use of the SeV can reprogram porcine somatic cells without first going through an intermediate pluripotent state. Our research produced piNSCs using SeV methods in novel, easily accessible large animal cell culture models for evaluating the efficacy of iNSC-based clinical translation in human medicine. Additionally, our piNSCs are potentially applicable in disease modeling in pigs and regenerative therapies in veterinary medicine.

Keywords: Sendai virus, cell reprogramming, porcine, neurosphere, differentiation, induced neural stem cells 


\section{INTRODUCTION}

The remarkable discovery that differentiated cells can be completely reprogrammed into induced pluripotent stem cells (iPSCs) by viral-mediated transduction of exogenous transcription factors marks a significant breakthrough in regenerative medicine (1). iPSCs offer an infinite supply of differentiated cells for various purposes, including disease modeling in vitro, drug research, toxicity testing, and autologous cell-based therapy (2). The potential for patient-specific cells to be used in autologous cell-based treatments is intriguing due to the reduction of risk from systemic immune response and the transmission of diseases via cell and tissue transplantation from the individual. The generation of neural stem cells (NSCs) and neurons from iPSCs is one of the most clinically relevant cell types (3-5). Nevertheless, this process is complicated by several factors, including a lengthy, inefficient reprogramming and differentiation processes (6), heterogenous cell differentiation (7), the possibility of tumor development as a result of undifferentiated iPSCs surviving in the differentiated iPSC population (4), and genomic instability (8).

Alternatively, the forced expression of NSC transcription factors (9-11) or pluripotency transcription factors, encoding OCT4, SOX2, KLF4, and c-MYC (OSKM) (12-14), converts differentiated cells directly into induced neural stem cells (iNSCs) and induced neural progenitor cells (iNPCs). This approach is an appealing alternative to existing iPSC technology because it enables the production of patient-specific NSCs without passing through the pluripotent stage, thereby decreasing the tumorigenic risk $(15,16)$. Since the first mouse iNSCs (miNSCs) were established in 2011 (12), many studies have been published detailing the derivation of iNSCs from a variety of species, including rats (17), monkeys (18), and humans (18-20). Established iNSCs have numerous features in common with embryonic brain-derived NSCs, including morphology, self-renewal capacity, gene and protein expression profiles, epigenetic state, as well as functional multipotency in vitro and in vivo $(9,21,22)$. Additionally, when iNSCs are transplanted into animal models for up to 6 months, they can alleviate disease phenotypes and prevent developing tumors, thus demonstrating their therapeutic promise for neurological disorders (23).

Although iNSCs are a feasible, effective, and autologous source for medical applications, their therapeutic potential has yet to be fully explored. Porcine iNSCs (piNSCs) may serve as a disease model for human regenerative medicine, as pigs have been established as one of the most effective large animal models in biomedical research and are often regarded as a preferable alternative to rodent models (24-26). Furthermore, preclinical evaluation of stem cell transplantation using piNSCs and their differentiation cells may be utilized to determine the safety and efficacy of iNSCs prior to human trials. Importantly, piNSCs are an appealing cell source for investigating pig disease in veterinary medicine. However, no commercially available piNSCs and their neural differentiation exist for studying pig neurological diseases, such as Streptococcus suis infections (27) and Japanese encephalitis (28). Until now, only one group has reported success in generating piNPCs using episomal plasmids; they demonstrated that piNPCs retain the capacity to grow for an extended period of time and differentiate efficiently into neurons in vitro (29).

Although previous studies have established numerous methods for directly converting somatic cells into iNSCs, most of the investigations rely on integrating viral vectors (such as lentiviral or retroviral approaches) $(9,13,15,30,31)$. These methods may result in insertional mutagenesis and the persistence or reactivation of transgenes. Moreover, therapeutic translation of this technique will require a thorough safety evaluation of mutations that occurred during the reprogramming process, as well as a fast derivation and differentiation strategy (32). A Sendai virus $(\mathrm{SeV})$ vector can overcome these issues due to its single-stranded RNA virus propagating in the cytoplasm of infected cells that neither pass through a DNA phase nor integrate into the host genome, unlike other viruses. As a result, the risks of tumorigenesis are reduced throughout the reprogramming process (33). With the $\mathrm{SeV}$ delivery system in kits, researchers may easily transduce the desired cells with $\mathrm{SeV}$ carrying OSKM for reprogramming and quickly remove $\mathrm{SeV}$ and transgenes via a temperature change due to its temperature sensitivity. Recently, SeV-based vectors have been widely utilized to generate human integration-free iPSCs (34-37) and have been adapted for the generation of iNSCs from human and monkey postnatal and adult fibroblasts (18). However, the generation of piNSCs using the $\mathrm{SeV}$ has not been explored yet.

In this investigation, we generated piNSC lines from pig fibroblasts by utilizing an integration-free $\mathrm{SeV}$ approach. The piNSCs displayed typical features of NSCs, such as morphology, gene expression patterns, self-renewal capacity, and differentiation potential. We anticipate that piNSCs will serve as novel, easily accessible large animal cell culture models for evaluating the efficacy of iNSCbased clinical translation. This pig model will allow us to assess the ultimate feasibility of personalized cell-based therapies. Furthermore, our piNSCs might be useful for disease modeling in pigs. As a result, this discovery is beneficial for veterinary medicine and possibly translation to human medicine.

\section{MATERIALS AND METHODS}

\section{Ethics Statement}

The Institutional Animal Care and Use Committee at the Faculty of Veterinary Science, Mahidol University, Thailand, reviewed and approved the experimental animal used in this study (Approval ID: MUVS-2015-49).

\section{Cell Culture}

All chemical compounds and cell culture reagents were acquired from Sigma-Aldrich (St. Louis, MO, USA) or Thermo Fisher Scientific (Waltham, MA, USA), unless otherwise specified. All cells were incubated in a humidified $5 \% \mathrm{CO}_{2}$ incubator at $37^{\circ} \mathrm{C}$. 


\section{Generation of piNSCs}

A porcine tail was received from an authorized farm in Ratchaburi Province, Thailand. Porcine tail fibroblasts (PTFs) were extracted using standard procedures from the tail of a three-day-old crossbred piglet, with minor modifications (38). The PTFs were propagated in fibroblast medium (FM) containing DMEM-high glucose, $10 \%$ fetal bovine serum (cat. no. SV30160, Hyclone, Logan, UT, USA), 1\% antibiotic-antimycotic solution, and $1 \%$ GlutaMAX $^{\mathrm{TM}}$. All cells were grown on feeder-free culture systems (Matrigel-coated dishes or plates) throughout the piNSC generation process. The PTFs were reprogrammed utilizing the integration-free CytoTune ${ }^{\mathrm{TM}}$-iPS 2.0 Sendai Reprogramming Kit, carrying three vector preparations: polycistronic human KLF4-OCT3/4-SOX2 (KOS), human CMYC, and human KLF4 (Thermo Fisher Scientific) following the manufacturer's instructions, with modifications (Figure 1A). The PTFs (passages 3 ) were seeded on 6-well plates at a density of $1 \times 10^{4}$ cells $/ \mathrm{cm}^{2} 1$ day before viral transduction to achieve approximately $60 \%-70 \%$ confluency at the time of transduction. The PTFs were transfected with $\mathrm{SeV}$ at a multiplicity of infection of 5 in an FM for $24 \mathrm{~h}$. The next day, the culture medium containing the $\mathrm{SeV}$ was removed and replaced with a new FM. The following day, the medium was switched to the iNSC medium (iNSCM) comprising DMEM/F-12 and a neurobasal medium at a ratio of $1: 1$ supplemented with $2 \%$ B-27 supplement, $1 \% \mathrm{~N}-2$ supplement, $1 \%$ antibiotic-antimycotic solution, $1 \%$ GlutaMAX, $20 \mathrm{ng} / \mathrm{mL}$ human basic fibroblast growth factor (bFGF; R\&D Systems), and $10 \mathrm{ng} / \mathrm{mL}$ human epidermal growth factor (hEGF). Seven days after reprogramming, the cells were dissociated with a $0.25 \%$ trypsin- ethylenediaminetetraacetic acid (EDTA) solution and replaced on a Matrigel-coated plate in piNSCM. Then, the appearance of epithelium-like colonies was monitored, and the medium was changed daily. Colonies with epithelium-like morphology were large enough to be collected around days 16-21 and were transferred onto an in vitro fertilization one-well dish for expansion. Sub-culturing at a 1:5 ratio with Versene $\AA$ Solution was performed every 2-3 days for further experiments.

\section{Neurosphere Formation}

The formation of neurospheres was investigated for iNSCs by resuspending 10,000 cells per well in iNSCM in 96-well plates covered with poly (2-hydroxyethyl methacrylate). Every 2 days, a fresh medium was added to the suspension cultures. Neurospheres were counted using a light microscope seven days after the suspension and collected for further study.

\section{Differentiation of piNSCs}

To induce spontaneous neuronal differentiation, piNSCs (P20) were dissociated and re-plated into a 6-well dish or a 24 -well plate with Matrigel-coated at a density of $2 \times 10^{4}$ cells per $\mathrm{cm}^{2}$ in the neuronal differentiation medium (the piNSCM without bFGF and hEGF). The media was replaced every 2 days for 14 days. Phase-contrast image analysis was performed daily to monitor cell differentiation in each well. At days 0 (proliferating piNSCs) and 14 (neuronal differentiation), the cells were fixed with $4 \%$ paraformaldehyde for immunofluorescence analysis and manually detached for western blot analysis.

\section{Cell Proliferation}

The cells were seeded at a density of $1 \times 10^{4}$ cells per well in a 96-well plate and maintained for two days in an iNSCM. Cell proliferation was assessed using the Cell Counting Kit-8 (CCK8 ) assay at various time points during culture (at 12, 24, 36, and $48 \mathrm{~h}$ ). To evaluate cell proliferation, $10 \mu \mathrm{l}$ of the CCK- 8 reagent was applied to live cells in 96-well plates, and suspension NSCs $\left(100 \mu \mathrm{l} /\right.$ well) were incubated for $3 \mathrm{~h}$ at $37^{\circ} \mathrm{C}$ with $5 \% \mathrm{CO}_{2}$. The absorbance at $450 \mathrm{~nm}$ was used to determine the number of cells. The CCK8 standard curve was used to quantify the number of viable cells according to the Dojindo CCK- 8 procedure. Briefly, cells were seeded in 96-well plates at $2.5 \times 10^{4}, 5 \times 10^{4}, 10$ $\times 10^{4}, 20 \times 10^{4}, 40 \times 10^{4}$, and $80 \times 10^{4}$ cells/well. The wells containing only media (without cells) were used to determine the background. Then, $10 \mu \mathrm{l}$ of CCK 8 was added to each well, and the plates were incubated at $37^{\circ} \mathrm{C}$ for $3 \mathrm{~h}$. The CCK 8 standard curve was established by plotting the number of cells vs. the absorbance.

\section{Immunofluorescence and Imaging Analysis}

Immunofluorescence analysis was utilized to detect NSC markers and distinguish neuronal cell lineages. Samples were fixed with $4 \%$ paraformaldehyde in cold phosphate-buffered saline (PBS) at $37^{\circ} \mathrm{C}$ for $15 \mathrm{~min}$. Then, samples were permeabilized with $0.25 \%$ Triton-X 100 in $\mathrm{PBS}$ at $37^{\circ} \mathrm{C}$ for $10 \mathrm{~min}$ and incubated with a non-specific binding blocking solution (2\% bovine serum albumin in PBS) at $37^{\circ} \mathrm{C}$ for $1 \mathrm{~h}$. Samples were treated overnight at $4^{\circ} \mathrm{C}$ in the dark with primary antibodies and then for $1 \mathrm{~h}$ at $37^{\circ} \mathrm{C}$ with secondary antibodies, as shown in Table 1. The coverslips were mounted on glass slides with an antifade mounting medium with DAPI (Vectashield, Vector Laboratories, Burlingame, CA, USA), and visualized using a Leica DMi8 inverted fluorescence microscope equipped with a Leica DFC7000 camera and the TCS SP8 confocal microscope equipped with a DFC3000G camera (Leica Microsystems, Wetzlar, Germany). For each sample, at least $40 \mathrm{z}$-stacks with 0.6-0.7 $\mathrm{m}$ intervals were acquired. All images were analyzed using the Leica Application Suite X (LAS X) imaging or ImageJ (NIH, USA) software to detect single fluorescence intensity measurements, the number of fluorescence positive cells, and colocalization. The images were measured in 20 randomly selected fields on each slide at a magnification of $\times 200$. At least three slides were scanned for each group to determine the expression of these markers ( $n=3$ independent experiments). Data are presented as the mean fluorescence intensity value \pm SEM after background signal subtraction. The percentage of positive cells per total number was based on the number of fluorescence marker-positive cells and DAPI-positive cell numbers measured by DAPI nuclear staining using ImageJ.

\section{Western Blot Analysis}

The cells were lysed using sonication in a radioimmunoprecipitation assay buffer, and the total protein concentration was examined by a protein assay kit (BioRad Laboratory, Hercules, CA, USA). Western blot analysis 

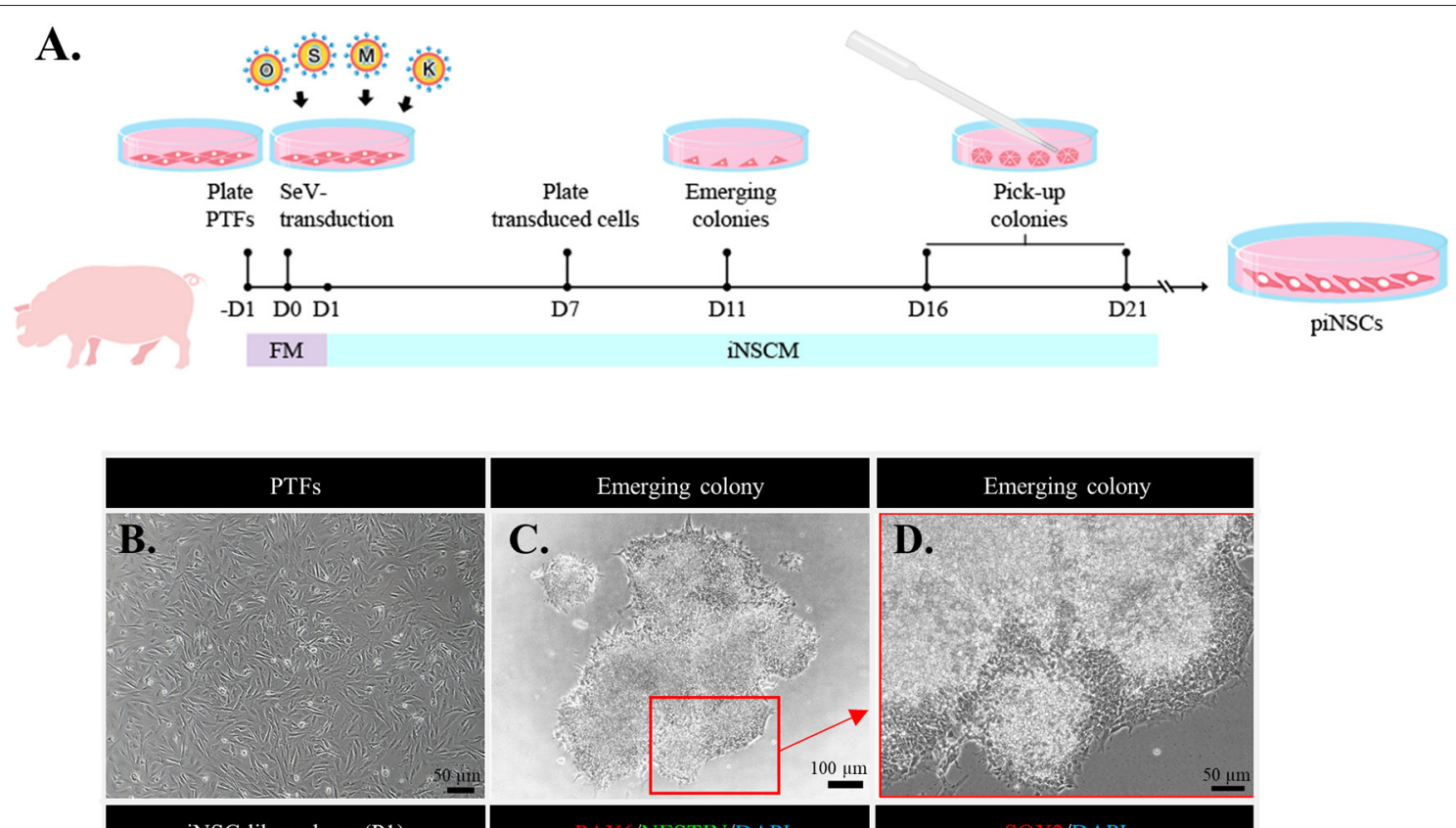

iNSC like colony (P1)
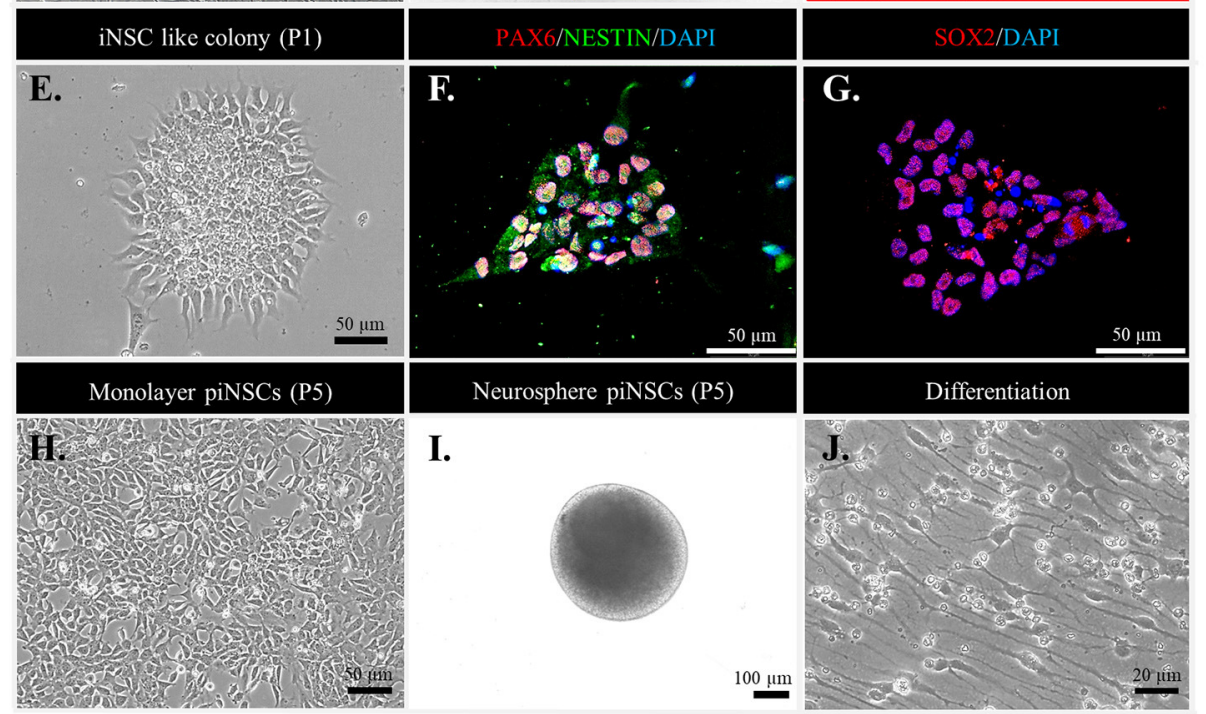

I.
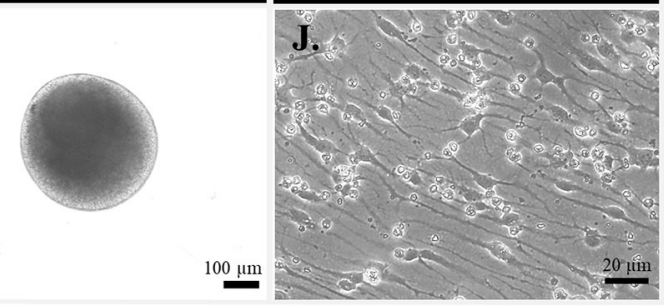

FIGURE 1 | Direct reprogramming of fibroblasts into porcine induced neural stem cell (piNSC) lines using an integration-free Sendai virus (SeV). (A) Schematic diagram illustrating the piNSC generation timeframe. (B) Phase-contrast image of porcine tail fibroblasts (PTFs) treated with SeV transfection overnight. (C) Phase-contrast image of an emerging piNSC-like colony 21 days after transfection (Passage 0; P0). The arrow indicates the extent to which the area of the cell in (C) was magnified in the high-resolution image (D). (D) High-resolution image of the cells in the inset in (C). (E) Phase-contrast image of piNSC-like colonies after first passage (P1), which are positive for early NSC markers PAX6/NESTIN [(F), red/green] and SOX2 [(G), red]. DAPI staining is blue. These colonies, which were positive for PAX6/NESTIN and SOX2, would be propagated to establish self-renewing piNSC lines. Morphology of iNSCs at P5 when cultured in a Matrigel-coated dish (H) and the formation of neurospheres when maintained in a suspension culture (I). piNSC-derived neurons showed typical neuronal morphology (J).

was performed using the Jess ${ }^{\mathrm{TM}}$ Simple Western system, an automated capillary-based size separation technique (ProteinSimple, San Jose, CA, USA) (39). The proteins of interest were separated and quantified using the $12-230-\mathrm{kDa}$ and 66-440-kDa Jess separation modules (SM-W004 and SM-W008, ProteinSimple) according to the manufacturer's instructions. The primary and secondary antibodies used in these studies are listed in Table 1. The chemiluminescent detection was achieved using peroxide/luminol-S (ProteinSimple). The chemiluminescent images of separated proteins in the capillary were acquired with Compass Simple Western version 5.0.1 software (Build 0911, Protein Simple) that automatically measured the area (chemiluminescence intensity). The results are shown as electropherograms reflecting the peak of the chemiluminescence intensity and as a lane view from the chemiluminescence signal detected in the capillary. The relative density was calculated by dividing the peak area of the protein of interest by the peak area of $\beta$-actin. 
TABLE 1 | List of antibodies used for immunofluorescence and western blot analysis.

\begin{tabular}{|c|c|c|c|c|}
\hline Markers & Antibodies & Dilution & Companies & Catalog number \\
\hline Cell proliferation & Rabbit anti-KI-67 & $1: 100$ & Santa cruz & sc-15402 \\
\hline \multirow[t]{3}{*}{ Pluripotency } & Goat anti-OCT4 & $1: 200$ & Santa cruz & sc-8628 \\
\hline & Goat anti-NANOG & $1: 100$ & R\&D system & AF 1997 \\
\hline & Mouse anti-SSEA1 & $1: 100$ & Santa cruz & sc-101462 \\
\hline \multirow[t]{4}{*}{ Neural stem cells } & Rabbit anti-PAX6 & $1: 100$ & Thermo fisher scientific & PA5-85374 \\
\hline & Rabbit anti-SOX2 & $1: 100$ & Santa cruz & sc-20088 \\
\hline & Mouse anti-NESTIN & $1: 100$ & Millipore & MAB5326 \\
\hline & Mouse anti-VIMENTIN & $1: 100$ & Santa cruz & sc-6260 \\
\hline \multirow[t]{6}{*}{ Neural lineage } & Rabbit anti-TUJ & $1: 100$ & Abcam & AB18207 \\
\hline & Mouse anti-MAP & $1: 100$ & Santa cruz & sc-32791 \\
\hline & Rabbit anti-GFAP & $1: 100$ & Millipore & AB5804 \\
\hline & Mouse anti-O4 & $1: 100$ & Millipore & MAB5804 \\
\hline & Mouse anti-SYP & $1: 100$ & Abcam & AB8049 \\
\hline & Rabbit anti-TH & $1: 100$ & Abcam & AB137869 \\
\hline Housekeeping protein & Rabbit anti- $\beta$-actin & $1: 500$ & Cell signaling & 4970s \\
\hline \multirow[t]{6}{*}{ Secondary antibodies } & Alexa Fluor 488 donkey anti-rabbit lgG & $1: 1,000$ & Thermo fisher scientific & A-21206 \\
\hline & Alexa Fluor 488 donkey anti-mouse lgG & $1: 1,000$ & Abcam & Ab150105 \\
\hline & Alexa Fluor 488 donkey anti-goat lgG & $1: 1,000$ & Thermo fisher scientific & A11055 \\
\hline & Alexa Fluor 594 donkey anti-rabbit lgG & $1: 1,000$ & Thermo fisher scientific & A-21207 \\
\hline & Alexa Fluor 594 donkey anti-mouse lgG & $1: 1,000$ & Thermo fisher scientific & A-21203 \\
\hline & Anti-mouse lgM-PE & $1: 1,000$ & Santa cruz & sc-3768 \\
\hline
\end{tabular}

\section{Karyotype Analysis}

The karyotype analysis followed a previously described procedure with minor modifications (40). Briefly, piNSC lines (P20) were cultured in a $6 \mathrm{~cm}$ culture dish at approximately $70 \%$ confluence and treated with a $5 \mu \mathrm{g} / \mathrm{mL}$ Colcemid solution (KaryoMAX ${ }^{\mathrm{TM}}$ Solution) for $1 \mathrm{~h}$ at $37^{\circ} \mathrm{C}$. The cells were gently dissociated using Versene ${ }^{\circledR}$ Solution and then treated for $15 \mathrm{~min}$ at $37^{\circ} \mathrm{C}$ with a hypotonic solution $(75 \mathrm{M} \mathrm{KCL})$. They were treated three times in a cold fixing solution (1:3 acetic acid to methanol concentration). The fixed cells were transferred to cool microscope slides and maintained at $37^{\circ} \mathrm{C}$ overnight. The slides were stained with Giemsa solution after soaking in a $0.05 \%$ trypsin EDTA solution at $37^{\circ} \mathrm{C}$. The images of 50 metaphases were captured by a Nikon Eclipse Ni with DS-Ri2 camera (Nikon Instruments, Japan) and analyzed by LUCIA Cytogenetics (Nikon Instruments, Japan).

\section{SeV Genome and Transgenes Analysis}

RT-PCR was used to evaluate the expression of the $\mathrm{SeV}$ genome and transgenes in both piNSCs. The RNeasy Mini Kit was used to lyse the cells and extract RNA (Genaid Biotech Ltd., New Taipei City, Taiwan). After that, $1 \mathrm{~g}$ of total RNA was reverse transcribed to cDNA using the SuperScript ${ }^{\mathrm{TM}}$ III First-Strand Synthesis System. In the PCR process, 50 ng template cDNA, 12.5L GoTaq PCR Master Mix (Promega, WI, USA), and 0.2 M of each primer were employed. PCR-amplified separation was achieved on $2 \%$ agarose gels and imaged using GelRed $($ nucleic acid staining (Biotium, Fremont, CA, USA). The relative expression levels of porcine endogenous genes of piNSC were also determined by RT-qPCR using the QuantiTect SYBR Green PCR Kit (QIAGEN,
Germany). The expression levels of each mRNA were normalized to those of the pig $\beta$-actin gene, a housekeeping control. Each 20- $\mu$ L PCR reaction consisted of 50-ng cDNA, $1 \times$ QuantiTect SYBR Green PCR master mix, and $0.2 \mu \mathrm{M}$ of each primer. The samples were incubated at $95^{\circ} \mathrm{C}$ for $15 \mathrm{~s}$, followed by 40 cycles of denaturation at $94^{\circ} \mathrm{C}$ for $15 \mathrm{~s}$, annealing at $60^{\circ} \mathrm{C}$ for $15 \mathrm{~s}$, and extension at $72^{\circ} \mathrm{C}$ for $30 \mathrm{~s}$. The threshold cycle $\left(\mathrm{C}_{\mathrm{T}}\right)$ values of endogenous genes and $\beta$-actin were used for relative quantification using the comparative $\mathrm{C}_{\mathrm{T}}\left(\Delta \Delta C_{\mathrm{T}}\right)$ method. The real-time data were analyzed using DataAssist software version 3.01 (Applied biosystems, Life Technologies Corp., NY, USA). Table 2 lists the primer sequences.

\section{Statistical Analysis}

Each experiment was conducted a minimum of three times. The quantitative results were expressed as the mean \pm standard error of the mean (SEM). The data was analyzed statistically using one-way analysis of variance for comparisons of more than two groups and the student's $t$-test for comparisons of the two groups. Additionally, Tukey's test was employed as a post hoc multiple comparison test for differences. All statistical analyses were carried out using SPSS version 25 software (IBM, USA). Statistical significance was defined as $p<0.05$.

\section{RESULTS}

\section{piNSC Lines Are Generated From PTFs Using the Non-integrative Sendai Virus}

To address the question of whether PTFs can be directly converted into stably expanding multipotent piNSCs, PTFs 
TABLE 2 | Primers used for reverse transcription polymerase chain reaction.

\begin{tabular}{|c|c|c|c|}
\hline \multirow[t]{2}{*}{ Genes } & \multicolumn{2}{|c|}{ Primer sequence $\left(5^{\prime}-3^{\prime}\right)$} & \multirow[t]{2}{*}{ Product size (bp) } \\
\hline & Forward & Reverse & \\
\hline \multicolumn{4}{|c|}{ Exogenous genes } \\
\hline $\mathrm{SeV}$ & GGA TCA CTA GGT GAT ATC GAG C & ACC AGA CAA GAG TIT AAG AGA TAT GTA TC & 181 \\
\hline hOCT4 & CCC GAA AGA GAA AGC GAA CCA G & AAT GTA TCG AAG GTG CTC AA & 483 \\
\hline hSOX2 & ATG CAC CGC TAC GAC GTG AGC GC & AAT GTA TCG AAG GTG CTC AA & 451 \\
\hline$h K L F 4$ & TTC CTG CAT GCC AGA GGA GCC C & AAT GTA TCG AAG GTG CTC AA & 410 \\
\hline$h C-M Y C$ & TAA CTG ACT AGC AGG CTT GTC G & TCC ACA TAC AGT CCT GGA TGA TG & 532 \\
\hline \multicolumn{4}{|c|}{ Endogenous genes } \\
\hline pOCT4 & ACA AGG AGA AGC TGG AGC CG & CGC GGA CCA CAT ССТ TCT CT & 752 \\
\hline pSOX2 & GGT TAC CTC TTC CCA CTC CA & CAA AAA TAG TCC CCC CAA AAG & 450 \\
\hline pNANOG & TCT GTG TCA GTT TGA GGG ACA GG & AAC AAG TAA AGC CTC CCT ATC CCA & 120 \\
\hline \multicolumn{4}{|c|}{ Oligodendrocyte gene } \\
\hline pMBP & GAG GCA GAG CTC CTG ACT ACA AA & GTC CCG TCC CAG CTT & 101 \\
\hline \multicolumn{4}{|c|}{ Housekeeping gene } \\
\hline$\beta$-actin & CGG GAC CTG ACT GAC TAC CTC & CCT TAA TGT CAC GCA CGA TIT CC & 93 \\
\hline
\end{tabular}

were isolated from the tail of a three-day-old, crossbred piglet (Large White/Landrace $\times$ Duroc). Non-integrative Sendai viral vectors carrying OSKM were transfected into PTFs for $24 \mathrm{~h}$ (Figure 1B). After that, the cells were cultured in FM for one day to recover and then in iNSCM for another day. On day 7 after transduction, the cells were dissociated and transferred onto Matrigel-coated 6-well plates in piNSCM at a density of $1 \times 10^{4}$ cells per well. On day 11 after transduction, iNSC clusters with neuroepithelial-like morphology emerged and developed quickly over the next week, revealing unclear and irregular boundaries with filament-like cells extending outward, which had a distinct morphology from that of porcine iPSC (piPSC) colonies. On days 16-21, the morphologically neuroepithelial-like colonies were mechanically triturated into small clusters and re-plated onto Matrigel-coated coverslips for immunostaining or onto a Matrigel-coated one-well dish for cell expansion in iNSCM (Figures 1C,D). The neuroepithelial colonies expressing early NSC markers such as PAX6, NESTIN, and SOX2 were continuously propagated using TrypLE Select Enzyme along the serial passaging for further characterization (Figures 1E-G). Hence, the transfection efficiency was $0.40 \%$, as measured by the number of neuroepithelial colonies expressing PAX6, NESTIN, and SOX2 divided by the total number of transfected cells. Additionally, immunofluorescence analysis revealed that the piNSC-like colonies (passage 2) were negative for the pluripotency markers OCT4 and NANOG, indicating that the colonies were not in a pluripotent state (Supplementary Figure 1). We generated a total of nine iNSC lines capable of proliferation in adherent monolayers (2D) (Figure 1H) or neurospheres (3D) (Figure 1I) as suspensiongrown neural cell aggregates and differentiation into neural lineages (Figure 1J). We used a pig tail to generate PTFs and iNSCs in this investigation. The generation of piNSCs was established in three replicates. We chose only two iNSC lines, namely VSMUi002-B and VSMUi002-E, based on their indefinite self-renewal potential and multipotency differentiation for further analysis. Each experiment involving two piNSC lines was performed at least three times.

\section{piNSC Lines Exhibit Characteristics of NSCs}

At passage 20, the piNSC lines (VSMUi002-B and VSMUi002E) displayed a neuroepithelial morphology in adherent monolayers (Figure 2A). The population cell doubling time of the VSMUi002-B and VSMUi002-E cell lines was approximately $24 \mathrm{~h}$, with no significant difference $(P>0.05)$ (Figure 2B). Both piNSC lines showed good proliferative capacity, as indicated by the high percentage of cells expressing KI67 $(75.3 \pm 1.18 \%$ and $78.6 \pm 1.19 \%$ ) (Figures 2A,C), and had been passaged more than 40 times. Hence, both iNSC lines had a strong capacity for self-renewal. They exhibited a typical diploid porcine karyotype (38, XX) during long-term culture (Figure 3A). Moreover, they displayed a high percentage of cells expressing the NSC markers, with nearly $100 \%$ of cells staining positive for PAX6, SOX2, NESTIN, and VIMENTIN, as determined by quantitative immunofluorescence analysis, which indicated the formation of a highly homogeneous population (Figures $\mathbf{2 A}, \mathbf{C}$ ). To corroborate the immunofluorescence results, endogenous PAX6, SOX2, VIMENTIN, and NESTIN proteins in piNSCs were quantified by western blot analysis in comparison with their parental PTFs. The expressions of PAX6 and SOX2 were substantially higher in VSMUi002-E than in VSMUi002-B. NESTIN expression was significantly higher in both piNSC lines than in PTFs. The PTFs lacked PAX6, SOX2, and NESTIN protein expression (Figure 3B; Supplementary Figure 3). On the other hand, the expression of VIMENTIN was higher in both piNSC lines than in PTFs (Figure 3B; Supplementary Figure 3). To investigate the pluripotent state, the piNSC lines (VSMUi002$B$ and VSMUi002-E) were analyzed using RT-PCR and immunofluorescence staining for pluripotency markers. RT-PCR analysis revealed that both piNSC lines were negative for pluripotency genes ( $p O C T 4$ and $p N A N O G)$ during early (P5, 
A
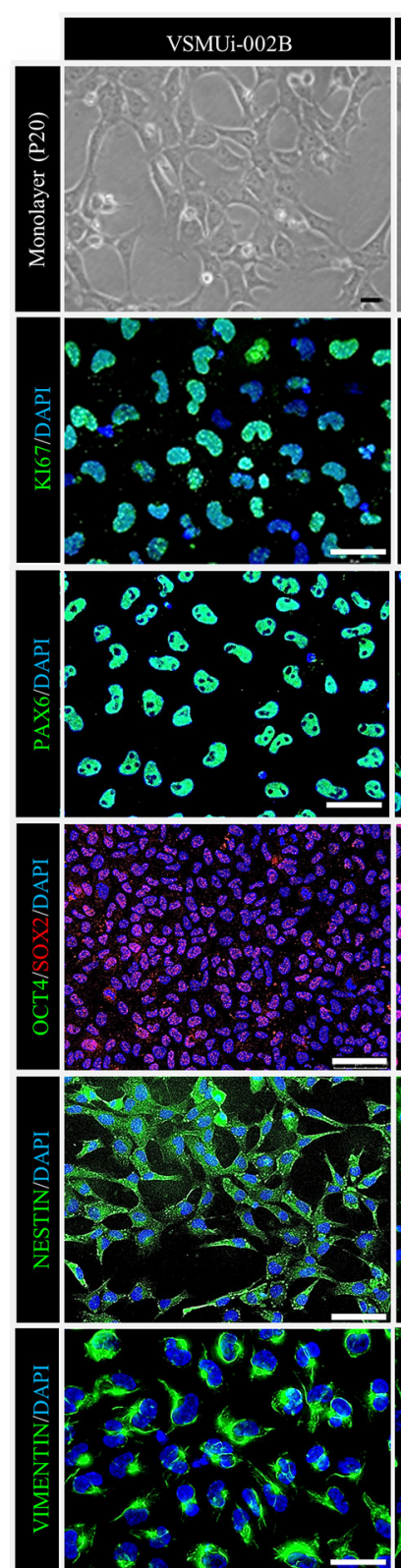

VSMUi-002E
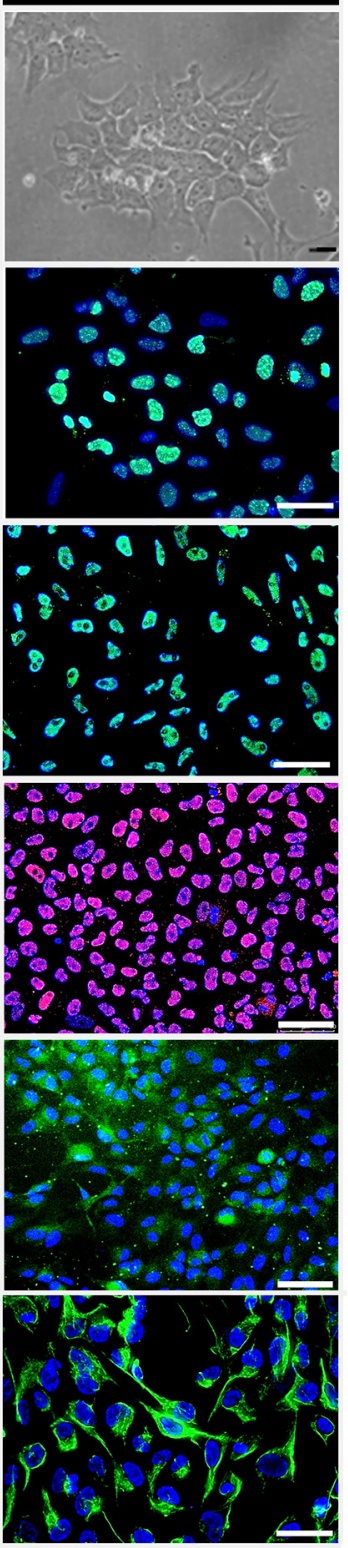

B
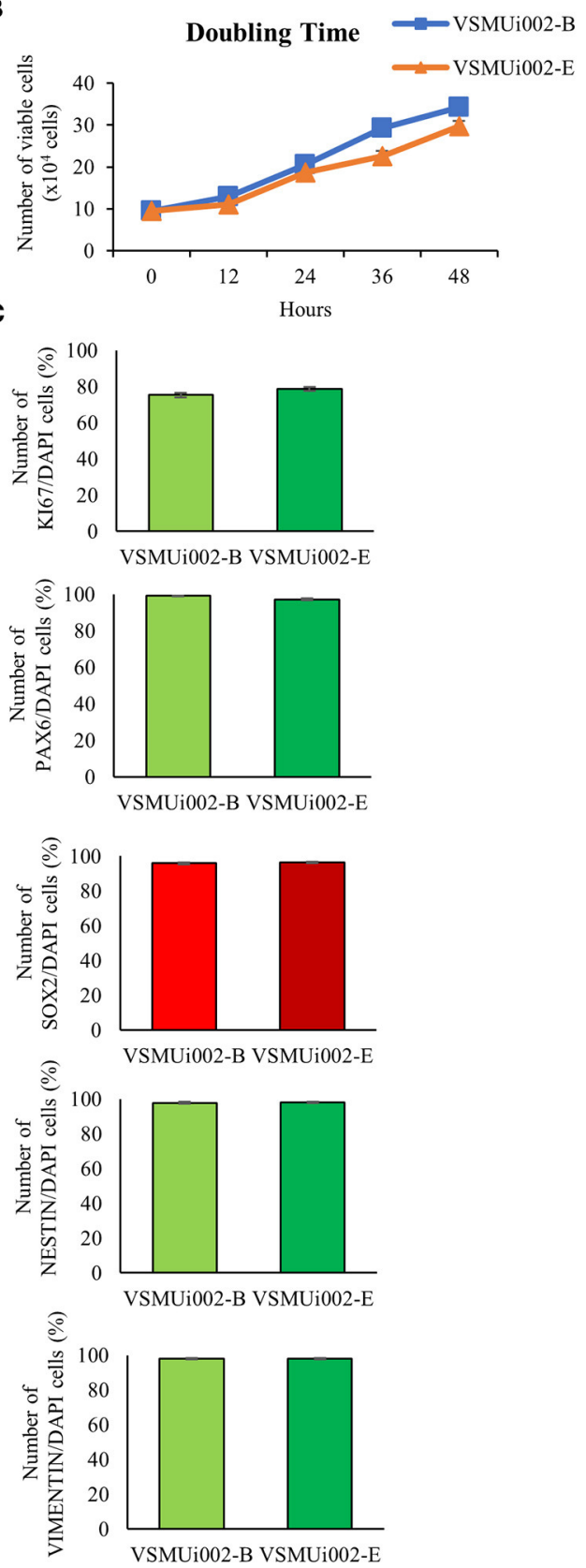

FIGURE 2 | Characterization of porcine induced neural stem cell (piNSC) lines. (A) At passage 20 (P20), the piNSCs had a neuroepithelial morphology and expressed the cell proliferation marker KI67 (green) as well as neural stem cell markers including PAX6 (green), SOX2 (red), NESTIN (green), and VIMENTIN (green), but not the pluripotency marker OCT4 (green). DAPI staining is blue. (B) The population cell doubling time of piNSC lines. (C) Quantitative evaluation of cell proliferation and neural stem cell markers in piNSC lines. Means with different lowercase letters are significantly different at $P<0.05$. Scale bars in (A) represent $10 \mu \mathrm{m}$.

P10, and P15) and late passages (P27, P30, and P33) (Figure 4A; Supplementary Figures 4, 5). Immunofluorescence staining revealed that both piNSC lines at passage 20 did not express pluripotency-related markers, including OCT4, NANOG, and SSEA1 (Figure 2A; Supplementary Figure 2). Hence, the piNSCs were not in a pluripotent state due to the absence of pluripotency markers. The piPSC line (VSMUi001-A) was used as a positive control to test for pluripotency markers (Supplementary Figure 2). To eliminate temperature-sensitive $\mathrm{SeV}$ vectors, piNSCs at passage 15 were grown at $39^{\circ} \mathrm{C}$ for viral inactivation, and cells were collected weekly for PCR detection of the residual virus. After 5 weeks of continuous culture, the residual virus was positive at passage 33, which indicated that $\mathrm{SeV}$ vectors were difficult to eliminate entirely. 
A

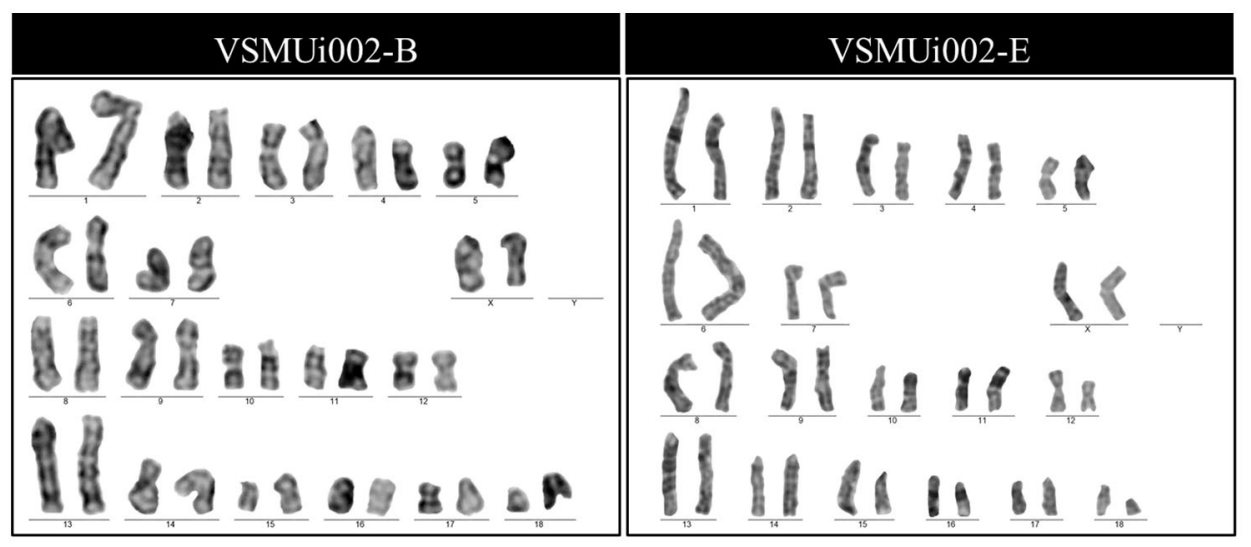

B
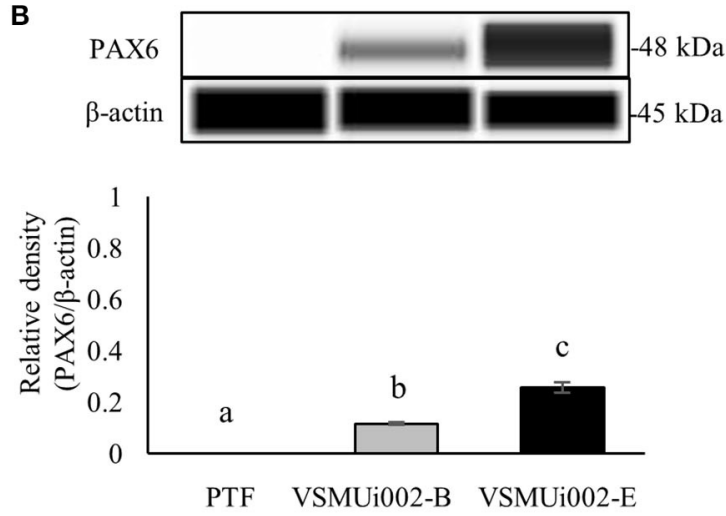

VIMENTIN

$\beta$-actin
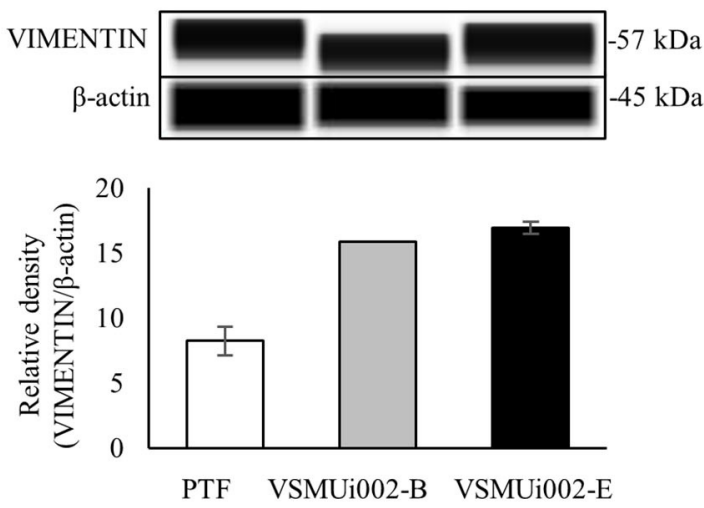
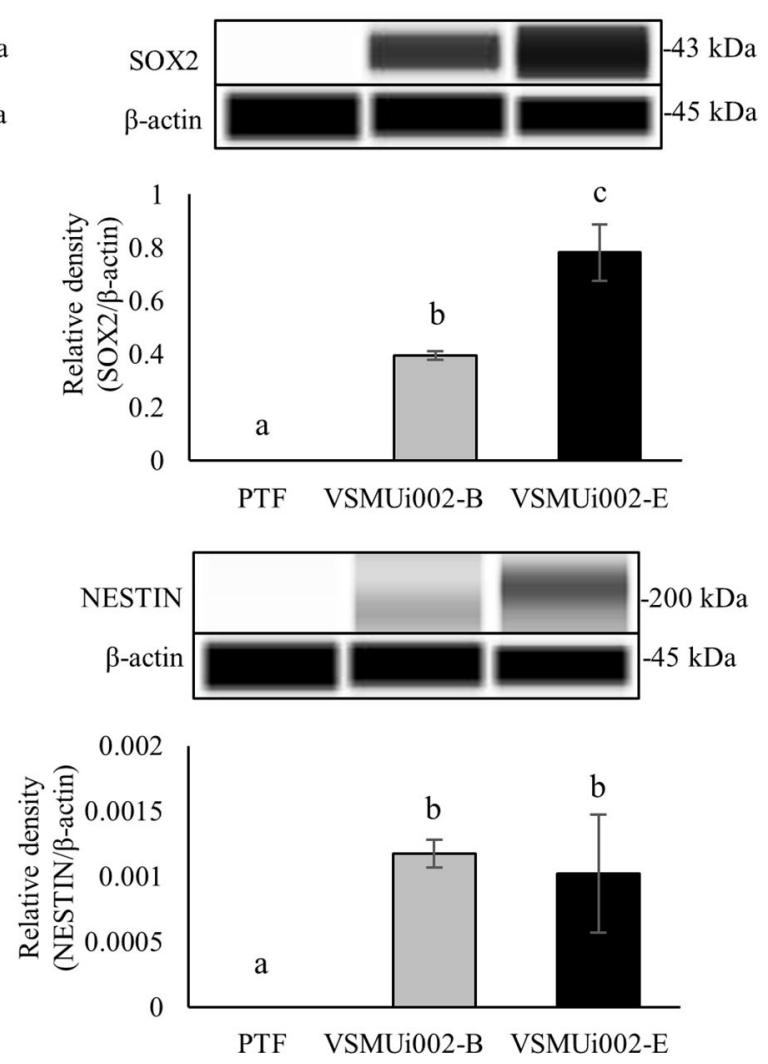

FIGURE 3 | Characterization of porcine induced neural stem cell (piNSC) lines (continuous). (A) Karyotype analysis of the piNSC lines showed a normal karyotype. (B) Western blot images of neural stem cell expressions (PAX6, SOX2, VIMENTIN, and NESTIN) and quantification of the western blot results. $\beta$-actin was used as an internal control. Means with different lowercase letters are significantly different at $P<0.05$.

We also excluded the irregular integration of SeV by comparing the amount of $\mathrm{SeV}$ in PTFs and piNSCs using real-time RTPCR, and found that the $\mathrm{SeV}$ expression was significantly decreased at $39^{\circ} \mathrm{C}$ (after the temperature change) compared to that at $37^{\circ} \mathrm{C}$ (Figure 4B). This finding implies that $\mathrm{SeV}$ did not integrate into the host genome. Moreover, piNSCs had undetectable levels of the three transgenes (hOCT4, hSOX2, and $h K L F 4)$, regardless of the temperature treatment, as determined by PCR. On the other hand, piNSCs remained positive for $h c-M Y C$, regardless of the temperature treatment (Figure 4A).

Both piNSC lines were able to form neurospheres (3D) in suspension cultures with similar efficiency, which were homogeneous in size and shape on day 7 (Figures 5A,B). Additionally, immunofluorescence labeling revealed that the neurospheres expressed NSC markers (PAX6, SOX2, 
A

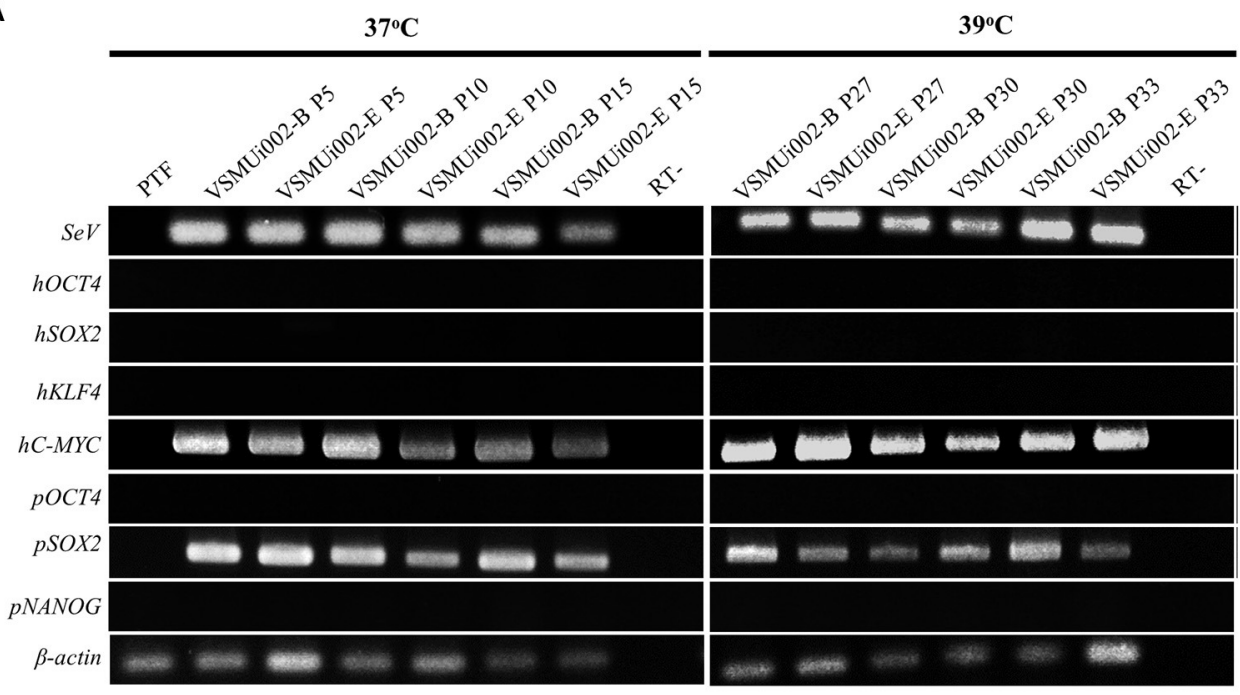

B

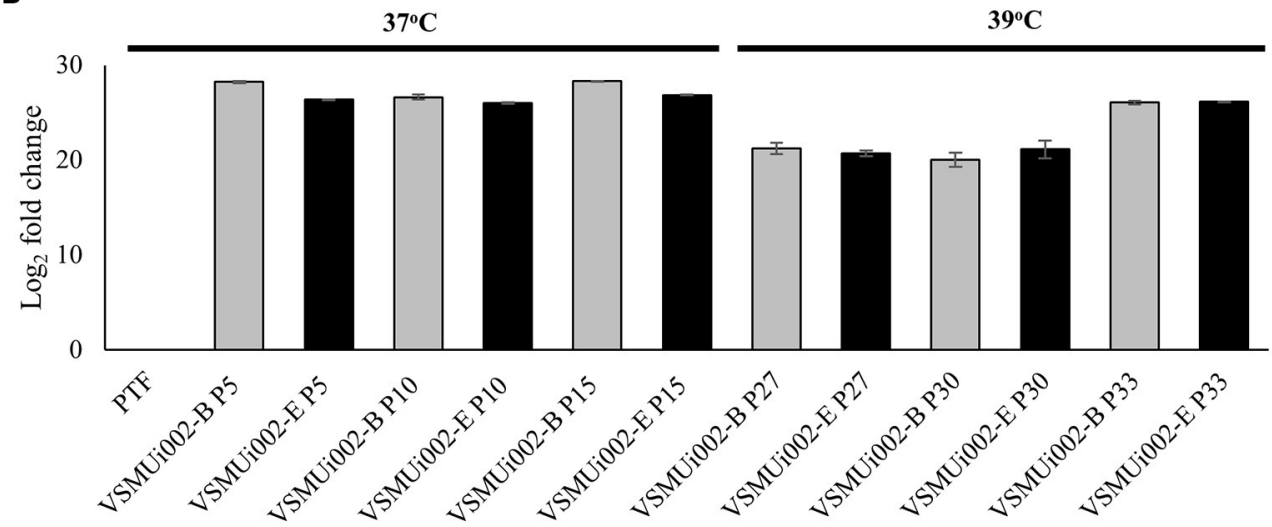

FIGURE 4 | Expression of exogenous and endogenous factors. (A) Expression of exogenous factors (hOCT4, hSOX2, hKLF4, hMYC), Sendai virus (SeV) RNA and endogenous factors (pOCT4, pSOX2, and pNANOG) was analyzed between 37 and $39^{\circ} \mathrm{C}$ for viral inactivation. (B) Fold change of SeV RNA expression in PTF and piNSCs at 37 and $39^{\circ} \mathrm{C}$ as determined by real time-PCR.

VIMENTIN) and a proliferation marker (KI67) (Figure 5C). Thus, piNSCs display neural progenitor features that can be obtained from the PTFs by $\mathrm{SeV}$ reprogramming.

\section{The piNSCs Spontaneously Differentiate to Neurons and Glia}

To determine the ability of piNSCs to differentiate spontaneously, they were dissociated into single cells and grown on a Matrigel substrate in a neural differentiation medium. After seven days of differentiation, piNSCs revealed significant morphological alterations, including a decrease in cell body size (Figure 6A), and expressed the immature neuronal marker (TUJ1) (Figure 6B). At day 14, the piNSCs exhibited mature neuronal morphology, including extensive and complex neurites (Figure 6A), which were positive for the mature neuronal marker (MAP2) (Figure 6B). The merged immunofluorescence images revealed a co-localization of
TUJ and MAP. Consistent with these findings, high levels of MAP2 protein expression colocalized with TUJ1 staining in VSMUi002-B and VSMUi002-E, with Pearson's correlation of $0.61 \pm 0.02$ and $0.79 \pm 0.01$, respectively. Additionally, some TUJ1-positive neurons were labeled with the synaptic protein synaptophysin (SYP) along neurites in a punctate manner (Figure 6B), which indicated possible synaptic connections. Furthermore, the piNSCs exhibited a high capacity for differentiation into dopamine-secreting neurons expressing tyrosine hydroxylase (TH) (Figure 6B). The piNSCs also developed into glial fibrillary acidic protein (GFAP)-positive astrocytes (Figure 6B). The staining intensities of all neuronalpositive cells (TUJ1, MAP2, TH, SYN, and GFAP) were the same in both iNSC lines (Figure 6C). Moreover, the RT-PCR analysis indicated an increase in myelin basic protein (MBP) expression, which is expressed mainly in oligodendrocytes (Figure 6D; Supplementary Figure 6), thus indicating the 
A

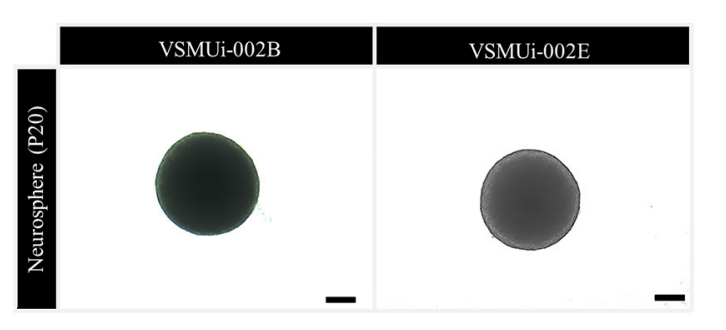

B

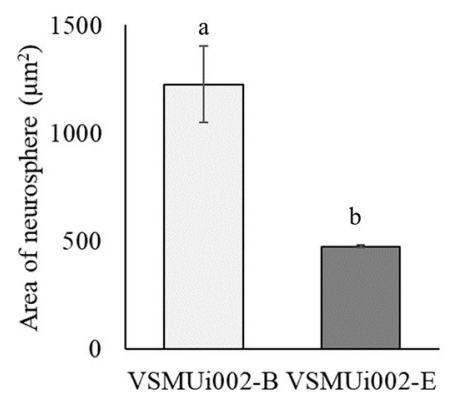

C

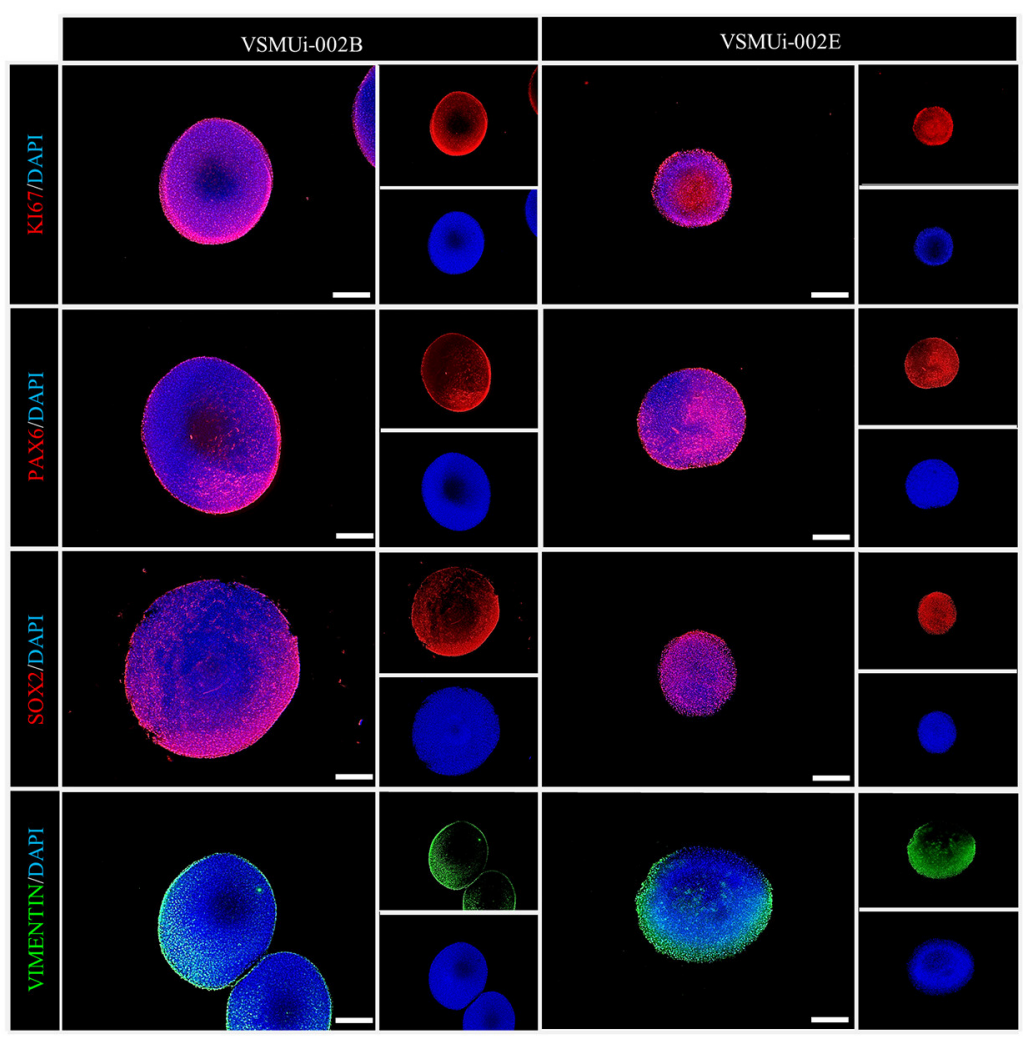

FIGURE 5 | Neurosphere formation of porcine induced neural stem cell (piNSC) lines. (A) Phase-contrast image of neurospheres on day 7 . (B) Average area of neurospheres. Means with different lowercase letters are significantly different at $P<0.05$. (C) Immunofluorescence staining of neurospheres exhibits the expression of the cell proliferation marker KI67 (red) as well as neural stem cell markers, including PAX6 (red), SOX2 (red), and VIMENTIN (green). The scale bars in (A) and (C) represent $100 \mu \mathrm{m}$.

existence of oligodendrocytes in differentiated derivatives. To corroborate the immunofluorescence results, endogenous TUJ1, MAP2, and GFAP proteins in iNSC-derived neural differentiation were quantified by western blot analysis in comparison with their parental iNSCs. The expression of these proteins was significantly higher in neuronal differentiation from VSMUi002-E than from VSMUi002-B (Figure 7; Supplementary Figure 7). Both iNSCs lacked MAP2 and GFAP protein expressions (Figure 7; Supplementary Figure 7). Interestingly, VSMUi002-E also expressed TUJ1 during the NSC stage (Figure 7; Supplementary Figure 7). Considered together, our data indicate that piNSCs have the capacity for multipotent neuronal differentiation.

\section{DISCUSSION}

Here, in comparison with prior studies, we explain several benefits of utilizing the temperature-sensitive $\mathrm{SeV}$ approach for reprogramming porcine fibroblasts into iNSCs: (1) Our piNSCs exhibit self-renewal and multipotency into neuronal and glial lineages without going through a pluripotent state, making them a safer alternative to piPSCs and (2) Stable and self-renewing 

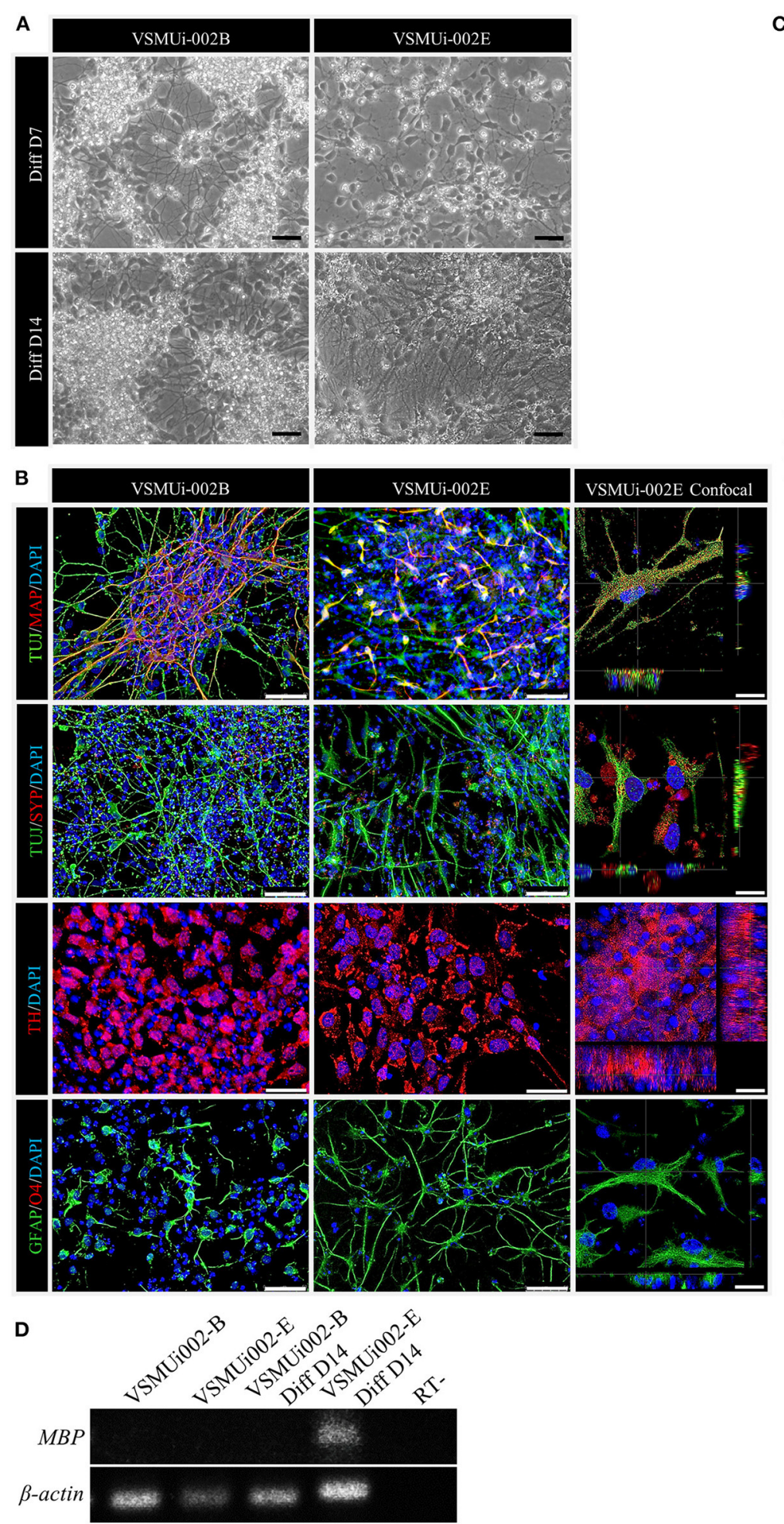

C
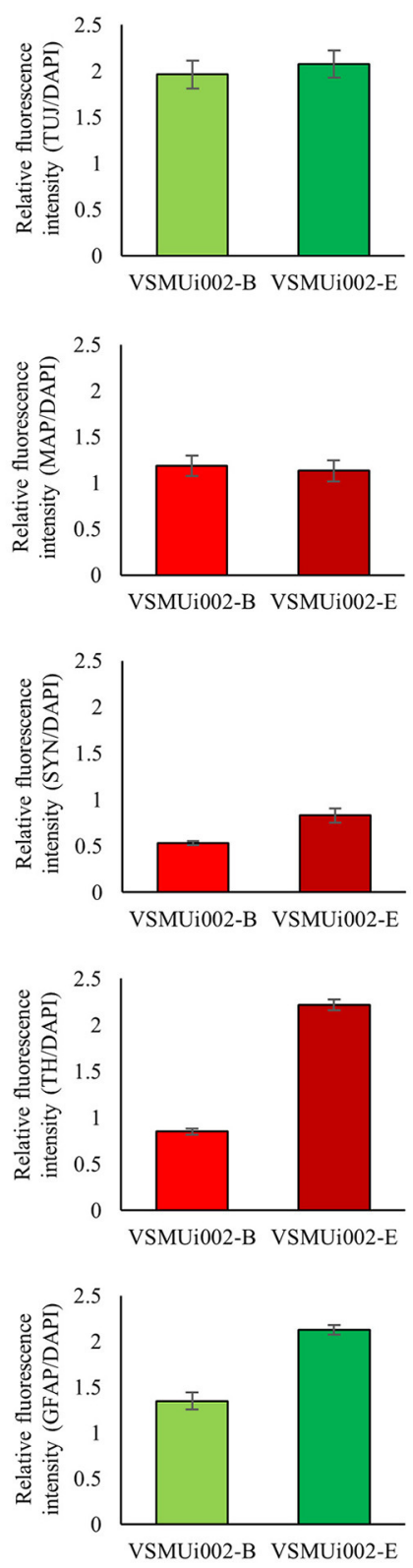

FIGURE 6 | In vitro differentiation potential of porcine induced neural stem cell (piNSC) lines. (A) Phase-contrast image of neural differentiation derived from piNSC lines on day 7 and day 14 of differentiation. (B) Neural differentiated from piNSC lines expressed immature neuronal marker (TUJ1, green), mature neuronal marker (MAP2, red), synaptic protein synaptophysin (SYP, red), dopamine-secreting neurons (TH, red), and astrocyte (GFAP, green). DAPI staining shown in blue. (C) Quantitative analysis of neurons and astrocytes derived from piNSC lines. The mean fluorescence signals for TUJ1, MAP2, SYP, TH, and GFAP were measured in 20 images per marker in each cell line under identical optical settings. (D) The VSMUi002-E piNSC line exhibited myelin basic protein (MBP), which is predominantly expressed in oligodendrocytes. The scale bars in (A) and (B) represent 20 and $50 \mu \mathrm{m}$, respectively. 


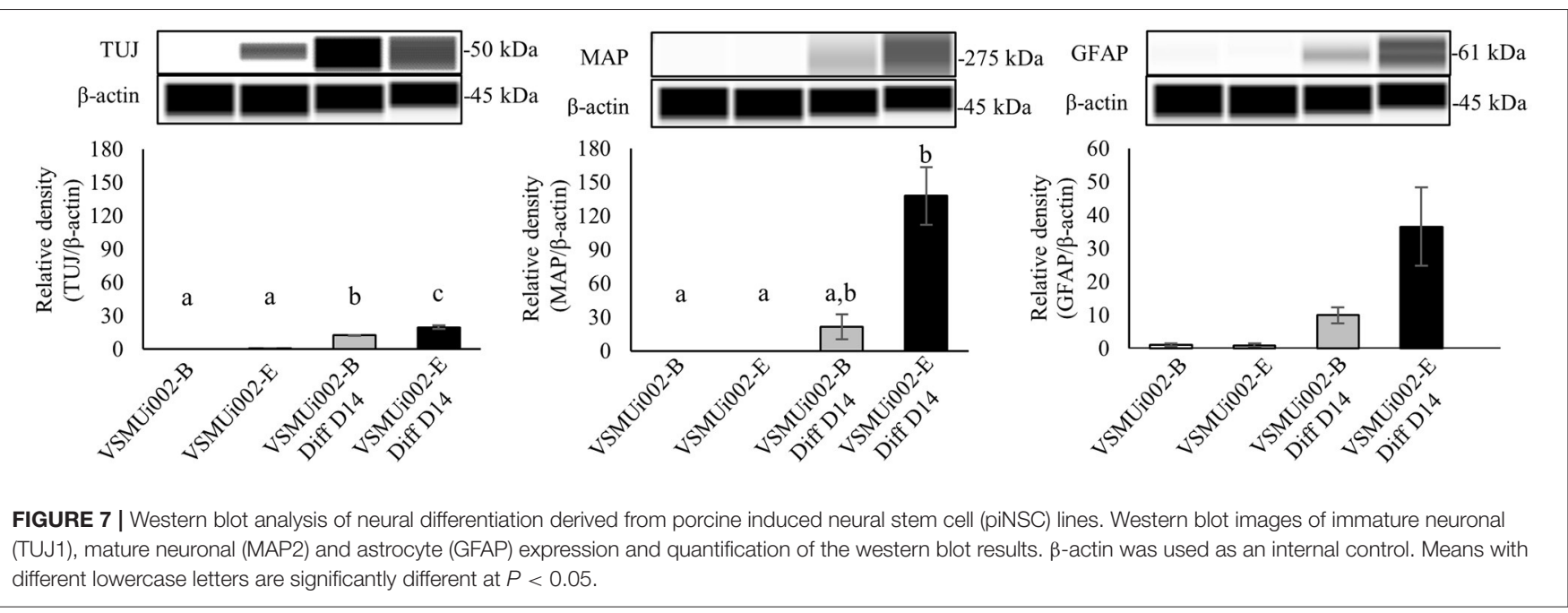

iNSCs can be generated from pig sources, which is the ultimate cell source for studying pig disease in veterinary medicine and may be advantageous for determining the efficacy of iNSCs prior to human trials (29).

The integration of techniques is frequently based on viral vector systems, which have been widely utilized and verified for the production of transgenic animal models and cell lines. Retroviral and lentiviral vectors are incorporated into the target genome, causing lasting genetic changes to the host genome and frequently affecting the transcriptome through persistent transgene expression $(9,13,15,30)$. Thus, while numerous studies have effectively transdifferentiated somatic cells into iNSCs using retro- or lentiviral vectors, a transgenic factor-free procedure is desirable to minimize the possibility of irreversible genetic alterations impairing the normal function of generated iNSCs. Alternatively, non-integrating methods for generating transgene-free iNSCs were devised, including the use of episomal vectors and the $\mathrm{SeV}$ in combination with or without small molecules $(19,41,42)$. The use of an episomal vector is inexpensive and easily adaptable for reprogramming a variety of cell types, including iPSCs and iNSCs. Episomal vectors are eliminated from these iPSCs via several passages through cell divisions, which can take several months to achieve transgenefree iPSCs $(30,32)$. However, $\mathrm{Xu}$ and colleagues indicated that piNPCs contained virtually undetectable amounts of EBNA1 , but episomal vector-transfected PFFs carried approximately 100 copies per cell, suggesting that EBNA-1 was not present in the piNPC genome (29). The $\mathrm{SeV}$ is a negative-sense singlestranded RNA virus that infects predominantly mammalian cells and replicates solely in their cytoplasm. In recent years, $\mathrm{SeV}$-based vectors have increasingly been utilized to generate transgene-free iPSCs and have been modified to generate iNSCs from human and monkey postnatal and adult fibroblasts (18, 43). In this study, piNSC lines were successfully produced for the first time utilizing an integration-free $\mathrm{SeV}$ method, with greater reprograming efficacy $(0.4 \%)$ than an integrationfree episomal approach (0.002\%) (29). In this study, we employed a commercially available reprogramming product, the temperature-sensitive $\mathrm{SeV}$ vector, which was designed to prevent replication at high temperatures, which results in the genome being passively eliminated during cell passage. The temperature-sensitive $\mathrm{SeV}$ vector is routinely used for generating human integration-free iNSC lines. Our findings indicated that the $\mathrm{SeV}$ vector and $h c-M Y C$ were expressed in piNSCs despite the high temperature-shift treatments that were utilized. The piNSC lines did not express hOCT4, $h S O X 2$, and $h K L F 4$, regardless of the temperature treatment. As previously described, the $\mathrm{SeV}$ vector is expressed and retained in the cytoplasm of piPSCs. The reason for the persistent expression of $\mathrm{SeV}$ and $h C-M Y C$ in our investigation is unclear. According to the Reprogramming Kit handbook, this might be because some cell strains (in this case, some species) take longer time to eliminate $\mathrm{SeV}$ and $\mathrm{C}-\mathrm{MYC}$ vectors and become vector-free. Similar to Congras and colleagues, they generated piPSCs using the $\mathrm{SeV}$ vector and revealed that while hOCT4 and hMYC expression were maintained throughout passages, $h S O X 2$ and $h K L F 4$ expression were undetectable following high temperature-shift treatments using RT-PCR. They demonstrated that exogenous factors are retained by the persistence and replication of the $\mathrm{SeV}$ genome in the cytoplasm of piPSCs rather than via genomic insertion (44). Similar to our result, $\mathrm{SeV}$ expression was significantly decreased after the temperature change.

Other integration-free systems rely on the usage of nonintegrating lentiviral vectors (integrase-defective lentiviral vectors), Protein, mRNAs and chemical-based. For a nonintegrating lentiviral vector system, a doxycycline-inducible lentivirus vector was recently modified to incorporate a loxPflanked expression cassette, enabling the creation of iNSCs. This allowed for later Cre-mediated transgene elimination following iNSC production. This lentivirus vector system can directly convert human adult monocytes, dermal, and fetal pancreatic fibroblasts into transgene-free iNSCs (45). TAT-SOX2 recombinant protein transduction combined with 
a cocktail of small chemical compounds to convert human adult fibroblasts directly into genetic material-free iNPCs (46). Additionally, mRNAs-encoding SOX2 reprogrammed human mesenchymal stem cells into integration-free iNSCs (47). Although the mRNA-based reprogramming method demonstrated a reduced risk of insertional mutagenesis of the host genome, this method has several limitations, including poor stability and being time-consuming (48). Direct conversion by chemical reprogramming has been shown to be up to $30 \%$ efficient. For instance, the production of iNSCs from mouse fibroblasts and human urine cells utilized a cocktail of three small chemicals: valproic acid (a histone deacetylase inhibitor), CHIR99021 (a GSK3 inhibitor), and RepSox (a TGFb inhibitor). Other research used a mixture of different pathway modulators to induce somatic cells to convert into iNSCs, including A-83-01 (a TGFb inhibitor), purmorphamine (a sonic hedgehog agonist), and thiazovivin (a ROCK inhibitor) (49, 50). Conversely, distinct cell populations were sensitive to pharmacologically active compounds that modulate cellular pathways involved in epigenetic status, transcriptional changes, cell signaling, and metabolism in order for chemical reprogramming to occur (51). The important limitation for chemical reprogramming is the risk of chemical-induced cell toxicity and genotoxicity as unintended side effects, which must be considered for each substance to design a reliable and robust system for direct conversion (52).

Our piNSC lines exhibited NSC markers (PAX6, SOX2, NESTIN, and VIMENTIN) and demonstrated self-renewal potential as determined by immunocytochemical labeling for proliferation markers KI67 in conjunction with essential markers for NSCs, which were similar to previous studies $(18,29,43)$. The $\mathrm{SeV}$ approach did not produce piPSC colonies with a high nucleus-to-cytoplasm ratio or clear colony borders, as previously described of piPSCs produced by viral vector-mediated methods $(26,53,54)$. Furthermore, the lack of OCT4, NANOG, and SSEA1 demonstrated that piNSC lines were not in a pluripotent state. As a result, piNSCs are considered to be safer than piPSCs $(15,16)$. Hence, the $\mathrm{SeV}$ provides an alternative reprogramming method that generates iNSCs without going through a pluripotent state. Our piNSCs are multipotent stem cells that are capable of generating neurons and glial cells, similar to previous findings $(29,55)$. However, the differentiation capacity varies according to the iNSC population, and the differentiation technique is utilized in vitro (e.g., spontaneous, undirected, and directed in vitro differentiation approaches) (45). Our findings confirmed that these piNSCs are more prone to developing into neurons and astroglia than into oligodendrocytes, since development into oligodendrocytes was less effective due to the short differentiation phase, which was consistent with a previous study (13).

\section{CONCLUSIONS}

Using the non-integration $\mathrm{SeV}$ for reprogramming, we effectively produced piNSC lines without going through an intermediate pluripotent stage. To our knowledge, this is the first attempt to use the $\mathrm{SeV}$ to directly reprogram somatic cells into NSCs in a porcine species. These piNSCs may provide an intriguing tool for determining the ultimate feasibility of disease modeling in pigs and cell-based regenerative treatment for human medicine. As a result, this finding is useful for veterinary medicine and the prospect of human medical translation.

\section{DATA AVAILABILITY STATEMENT}

The original contributions presented in the study are included in the article/Supplementary Material, further inquiries can be directed to the corresponding author.

\section{ETHICS STATEMENT}

The animal study was reviewed and approved by the Institutional Animal Care and Use Committee at Faculty of Veterinary Science, Mahidol University, Thailand (Approval ID: MUVS2015-49).

\section{AUTHOR CONTRIBUTIONS}

WC and SR were responsible for funding acquisition, conceptualization, original draft manuscript writing, execution of the majority of experiments, including the establishment and characterization of piNSCs. RR was responsible for the initial cell reprogramming process. LS was responsible for the molecular analysis. PJ, NC, and SC conducted cell culture, immunofluorescence, and imaging analyses. WC analyzed the data. JF and SR contributed to the review and editing of the final manuscript. All authors approved the final version submitted for publication.

\section{FUNDING}

This research has received funding support from the NSRF via the Program Management Unit for Human Resources \& Institutional Development, Research and Innovation [Grant number B05F630046] and Mahidol University [Basic Research Fund: fiscal year 2021].

\section{ACKNOWLEDGMENTS}

We would like to express our gratitude to the following experts and institutions for their contributions: Thanks to Prof. Dr. Kamthorn Pruksananonda and Pranee Numchaisrika from the Department of Obstetrics and Gynecology, Faculty of Medicine, Chulalongkorn University, for the equipment provision and technical assistance. Additionally, we would like to thank Sirikron Pamonsupornvichit for doing the karyotype analysis and Wipawee Pavarajarn for assisting with the cell reprogramming process. Furthermore, we would like to thank Supath Tantivitayamas and Onvara Ritudomphol from Histocenter Thailand Co., Ltd. for providing a Leica DMi8 
inverted fluorescent microscope equipped with a Leica DFC7000 camera, Utarat Imsaard from Hollywood International Ltd. for providing a DS-Ri2 camera and the LUCIA Cytogenetics System, and Nawapol Udpuay and staff of Mahidol UniversityFrontier Research Facility (MU-FRF), Mahidol University, for providing the flow cytometry machine and Jess automated western blotting. Finally, we would like to express our deepest gratitude to the MoZWE, Faculty of Veterinary Science, Mahidol

\section{REFERENCES}

1. Takahashi K, Yamanaka S. Induction of pluripotent stem cells from mouse embryonic and adult fibroblast cultures by defined factors. Cell. (2006) 126:663-76. doi: 10.1016/j.cell.2006.07.024

2. Moradi S, Mahdizadeh H, Saric T, Kim J, Harati J, Shahsavarani H, et al. Research and therapy with induced pluripotent stem cells (iPSCs): social, legal, and ethical considerations. Stem Cell Res Ther. (2019) 10:341. doi: 10.1186/s13287-019-1455-y

3. Du ZW, Chen H, Liu H, Lu J, Qian K, Huang CL, et al. Generation and expansion of highly pure motor neuron progenitors from human pluripotent stem cells. Nat Commun. (2015) 6:6626. doi: 10.1038/ncomms7626

4. Lopez-Serrano C, Torres-Espin A, Hernandez J, Alvarez-Palomo AB, Requena J, Gasull X, et al. Effects of the post-spinal cord injury microenvironment on the differentiation capacity of human neural stem cells derived from induced pluripotent stem cells. Cell Transplant. (2016) 25:1833-52. doi: 10.3727/096368916X691312

5. Lu J, Zhong X, Liu H, Hao L, Huang CT, Sherafat MA, et al. Generation of serotonin neurons from human pluripotent stem cells. Nat Biotechnol. (2016) 34:89-94. doi: 10.1038/nbt.3435

6. Hu BY, Weick JP, Yu J, Ma LX, Zhang XQ, Thomson JA, et al. Neural differentiation of human induced pluripotent stem cells follows developmental principles but with variable potency. Proc Natl Acad Sci USA. (2010) 107:4335-40. doi: 10.1073/pnas.0910012107

7. Chambers SM, Fasano CA, Papapetrou EP, Tomishima M, Sadelain M, Studer L. Highly efficient neural conversion of human ES and iPS cells by dual inhibition of SMAD signaling. Nat Biotechnol. (2009) 27:275-80. doi: 10.1038/nbt.1529

8. Weissbein U, Ben-David U, Benvenisty N. Virtual karyotyping reveals greater chromosomal stability in neural cells derived by transdifferentiation than those from stem cells. Cell Stem Cell. (2014) 15:687-91. doi: $10.1016 /$ j.stem.2014.10.018

9. Han DW, Tapia N, Hermann A, Hemmer K, Hoing S, Arauzo-Bravo MJ, et al. Direct reprogramming of fibroblasts into neural stem cells by defined factors. Cell Stem Cell. (2012) 10:465-72. doi: 10.1016/j.stem.2012.02.021

10. Sheng C, Zheng Q, Wu J, Xu Z, Wang L, Li W, et al. Direct reprogramming of Sertoli cells into multipotent neural stem cells by defined factors. Cell Res. (2012) 22:208-18. doi: 10.1038/cr.2011.175

11. Lujan E, Chanda S, Ahlenius H, Sudhof TC, Wernig M. Direct conversion of mouse fibroblasts to self-renewing, tripotent neural precursor cells. Proc Natl Acad Sci USA. (2012) 109:2527-32. doi: 10.1073/pnas.1121003109

12. Kim J, Efe JA, Zhu S, Talantova M, Yuan X, Wang S, et al. Direct reprogramming of mouse fibroblasts to neural progenitors. Proc Natl Acad Sci USA. (2011) 108:7838-43. doi: 10.1073/pnas.1103113108

13. Thier M, Worsdorfer P, Lakes YB, Gorris R, Herms S, Opitz T, et al. Direct conversion of fibroblasts into stably expandable neural stem cells. Cell Stem Cell. (2012) 10:473-9. doi: 10.1016/j.stem.2012.03.003

14. Cairns DM, Chwalek K, Moore YE, Kelley MR, Abbott RD, Moss S, et al. Expandable and rapidly differentiating human induced neural stem cell lines for multiple tissue engineering applications. Stem Cell Reports. (2016) 7:55770. doi: 10.1016/j.stemcr.2016.07.017

15. Ring KL, Tong LM, Balestra ME, Javier R, Andrews-Zwilling Y, Li G, et al. Direct reprogramming of mouse and human fibroblasts into multipotent neural stem cells with a single factor. Cell Stem Cell. (2012) 11:100-9. doi: 10.1016/j.stem.2012.05.018
University, and their staff for providing laboratory space and technical help.

\section{SUPPLEMENTARY MATERIAL}

The Supplementary Material for this article can be found online at: https://www.frontiersin.org/articles/10.3389/fvets. 2021.806785/full\#supplementary-material

16. Gao M, Yao H, Dong Q, Zhang H, Yang Z, Yang Y, et al. Tumourigenicity and immunogenicity of induced neural stem cell grafts versus induced pluripotent stem cell grafts in syngeneic mouse brain. Sci Rep. (2016) 6:29955. doi: 10.1038/srep29955

17. Xi G, Hu P, Qu C, Qiu S, Tong C, Ying QL. Induced neural stem cells generated from rat fibroblasts. Genomics Proteomics Bioinformatics. (2013) 11:312-9. doi: 10.1016/j.gpb.2013.09.003

18. Lu J, Liu H, Huang CT, Chen H, Du Z, Liu Y, et al. Generation of integrationfree and region-specific neural progenitors from primate fibroblasts. Cell Rep. (2013) 3:1580-91. doi: 10.1016/j.celrep.2013.04.004

19. Wang L, Wang L, Huang W, Su H, Xue Y, Su Z, et al. Generation of integration-free neural progenitor cells from cells in human urine. Nat Methods. (2013) 10:84-9. doi: 10.1038/nmeth.2283

20. Kwak TH, Hali S, Kim S, Kim J, La H, Kim KP, et al. Robust and reproducible generation of induced neural stem cells from human somatic cells by defined factors. Int J Stem Cells. (2020) 13:80-92. doi: 10.15283/ijsc 19097

21. Kim SM, Kim JW, Kwak TH, Park SW, Kim KP, Park H, et al. Generation of integration-free induced neural stem cells from mouse fibroblasts. J Biol Chem. (2016) 291:14199-212. doi: 10.1074/jbc.M115.713578

22. Yao H, Gao M, Ma J, Zhang M, Li S, Wu B, et al. Transdifferentiation-induced neural stem cells promote recovery of middle cerebral artery stroke rats. PLoS ONE. (2015) 10:e0137211. doi: 10.1371/journal.pone.0137211

23. Hemmer K, Zhang M, van Wullen T, Sakalem M, Tapia N, Baumuratov A, et al. Induced neural stem cells achieve long-term survival and functional integration in the adult mouse brain. Stem Cell Reports. (2014) 3:423-31. doi: 10.1016/j.stemcr.2014.06.017

24. Harding J, Roberts RM, Mirochnitchenko O. Large animal models for stem cell therapy. Stem Cell Res Ther. (2013) 4:23. doi: 10.1186/scrt171

25. Xu J, Yu L, Guo J, Xiang J, Zheng Z, Gao D, et al. Generation of pig induced pluripotent stem cells using an extended pluripotent stem cell culture system. Stem Cell Res Ther. (2019) 10:193. doi: 10.1186/s13287-019-1303-0

26. Chakritbudsabong W, Chaiwattanarungruengpaisan S, Sariya L, Pamonsupornvichit S, Ferreira JN, Sukho P, et al. Exogenous LIN28 Is required for the maintenance of self-renewal and pluripotency in presumptive porcine-induced pluripotent stem cells. Front Cell Dev Biol. (2021) 9:709286. doi: 10.3389/fcell.2021.709286

27. Zheng P, Zhao YX, Zhang AD, Kang C, Chen HC, Jin ML. Pathologic analysis of the brain from Streptococcus suis type 2 experimentally infected pigs. Vet Pathol. (2009) 46:531-5. doi: 10.1354/vp.08-VP-0043-J-FL

28. Yamada M, Nakamura K, Yoshii M, Kaku Y. Nonsuppurative encephalitis in piglets after experimental inoculation of Japanese encephalitis flavivirus isolated from pigs. Vet Pathol. (2004) 41:62-7. doi: 10.1354/vp.41-1-62

29. Xu XL, Yang JP, Fu LN, Ren RT, Yi F, Suzuki K, et al. Direct reprogramming of porcine fibroblasts to neural progenitor cells. Protein Cell. (2014) 5:4-7. doi: 10.1007/s13238-013-0015-y

30. Lee M, Ha J, Son YS, Ahn H, Jung KB, Son MY, et al. Efficient exogenous DNAfree reprogramming with suicide gene vectors. Exp Mol Med. (2019) 51:1-12. doi: 10.1038/s12276-019-0299-y

31. Shahbazi E, Moradi S, Nemati S, Satarian L, Basiri M, Gourabi H, et al. Conversion of human fibroblasts to stably self-renewing neural stem cells with a single zinc-finger transcription factor. Stem Cell Reports. (2016) 6:539-51. doi: 10.1016/j.stemcr.2016.02.013

32. Yuan Y, Tang X, Bai YF, Wang S, An J, Wu Y, et al. Dopaminergic precursors differentiated from human blood-derived induced neural stem 
cells improve symptoms of a mouse Parkinson's disease model. Theranostics. (2018) 8:4679-94. doi: 10.7150/thno.26643

33. Nakanishi M, Otsu M. Development of Sendai virus vectors and their potential applications in gene therapy and regenerative medicine. Curr Gene Ther. (2012) 12:410-6. doi: 10.2174/156652312802762518

34. Fusaki N, Ban H, Nishiyama A, Saeki K, Hasegawa M. Efficient induction of transgene-free human pluripotent stem cells using a vector based on Sendai virus, an RNA virus that does not integrate into the host genome. Proc Jpn Acad Ser B Phys Biol Sci. (2009) 85:348-62. doi: 10.2183/pjab.85.348

35. Seki $T$, Yuasa $S$, Oda $M$, Egashira $T$, Yae $K$, Kusumoto $D$, et al. Generation of induced pluripotent stem cells from human terminally differentiated circulating $\mathrm{T}$ cells. Cell Stem Cell. (2010) 7:11-4. doi: $10.1016 /$ j.stem.2010.06.003

36. Ban H, Nishishita N, Fusaki N, Tabata T, Saeki K, Shikamura M, et al. Efficient generation of transgene-free human induced pluripotent stem cells (iPSCs) by temperature-sensitive Sendai virus vectors. Proc Natl Acad Sci USA. (2011) 108:14234-9. doi: 10.1073/pnas.1103509108

37. Tongkobpetch S, Rungsiwiwut R, Pruksananonda K, Suphapeetiporn K, Shotelersuk V. Generation of two human iPSC lines (MDCUi001-A and MDCUi001-B) from dermal fibroblasts of a Thai patient with X-linked osteogenesis imperfecta using integration-free Sendai virus. Stem Cell Res. (2019) 39:101493. doi: 10.1016/j.scr.2019.101493

38. Khan $M$, Gasser $S$. Generating primary fibroblast cultures from mouse ear and tail tissues. J Vis Exp. (2016):53565. doi: 10.3791/53565

39. Edouard S, Jaafar R, Orain N, Parola P, Colson P, La Scola B, et al. Automated Western immunoblotting detection of anti-SARS-CoV2 serum antibodies. Eur J Clin Microbiol Infect Dis. (2021) 40:1309-17. doi: 10.1007/s10096-021-04203-8

40. Phakdeedindan P, Setthawong P, Tiptanavattana N, Rungarunlert S, Ingrungruanglert $\mathrm{P}$, Israsena $\mathrm{N}$, et al. Rabbit induced pluripotent stem cells retain capability of in vitro cardiac differentiation. Exp Anim. (2019) 68:35-47. doi: 10.1538/expanim.18-0074

41. Tang X, Wang S, Bai Y, Wu J, Fu L, Li M, et al. Conversion of adult human peripheral blood mononuclear cells into induced neural stem cell by using episomal vectors. Stem Cell Res. (2016) 16:236-42. doi: $10.1016 / j . s c r .2016 .01 .016$

42. Kim SM, Flasskamp H, Hermann A, Arauzo-Bravo MJ, Lee SC, Lee SH, et al. Direct conversion of mouse fibroblasts into induced neural stem cells. Nat Protoc. (2014) 9:871-81. doi: 10.1038/nprot.2014.056

43. Meyer S, Wörsdörfer P, Günther K, Thier M, Edenhofer F. Derivation of adult human fibroblasts and their direct conversion into expandable neural progenitor cells. J Vis Exp. (2015):e52831. doi: 10.3791/52831

44. Congras A, Barasc H, Canale-Tabet K, Plisson-Petit F, Delcros C, Feraud O, et al. Corrigendum: non integrative strategy decreases chromosome instability and improves endogenous pluripotency genes reactivation in porcine induced pluripotent-like stem cells. Sci Rep. (2018) 8:46931. doi: 10.1038/srep 46931

45. Thier MC, Hommerding O, Panten J, Pinna R, Garcia-Gonzalez D, Berger $\mathrm{T}$, et al. Identification of embryonic neural plate border stem cells and their generation by direct reprogramming from adult human blood cells. Cell Stem Cell. (2019) 24:1-17. doi: 10.1016/j.stem.2018.11.015

46. Mirakhori F, Zeynali B, Rassouli H, Shahbazi E, Hashemizadeh S, Kiani S, et al. Induction of neural progenitor-like cells from human fibroblasts via a genetic material-free approach. PLoS ONE. (2015) 10:1-17. doi: 10.1371/journal.pone. 0135479

47. Kim B, Choi SW, Shin J, Kim J, Kang I, Lee B, et al. Single-factor SOX2 mediates direct neural reprogramming of human mesenchymal stem cells via transfection of in vitro transcribed mRNA. Cell Transplant. (2018) 27:115467. doi: 10.1177/0963689718771885

48. Steinle H, Behring A, Schlensak C, Wendel HP, Avci-Adali M. Concise review: application of in vitro transcribed messenger RNA for cellular engineering and reprogramming: progress and challenges. Stem Cells. (2017) 35:68-79. doi: $10.1002 /$ stem.2402

49. Zhang M, Lin YH, Sun YJ, Zhu S, Zheng J, Liu K, et al. Pharmacological reprogramming of fibroblasts into neural stem cells by signalingdirected transcriptional activation. Cell Stem Cell. (2016) 18:653-67. doi: 10.1016/j.stem.2016.03.020

50. Zheng J, Choi KA, Kang PJ, Hyeon S, Kwon S, Moon JH, et al. A combination of small molecules directly reprograms mouse fibroblasts into neural stem cells. Biochem Biophys Res Commun. (2016) 476:42-8. doi: 10.1016/j.bbrc.2016.05.080

51. Xie X, Fu Y, Liu J. Chemical reprogramming and transdifferentiation. Curr Opin Genet Dev. (2017) 46:104-13. doi: 10.1016/j.gde.2017.07.003

52. Langie SA, Koppen G, Desaulniers D, Al-Mulla F, Al-Temaimi R, Amedei A, et al. Causes of genome instability: the effect of low dose chemical exposures in modern society. Carcinogenesis. (2015) 36 (Suppl 1):S61-88. doi: 10.1093/carcin/bgv031

53. Esteban MA, Xu J, Yang J, Peng M, Qin D, Li W, et al. Generation of induced pluripotent stem cell lines from Tibetan miniature pig. J Biol Chem. (2009) 284:17634-40. doi: 10.1074/jbc.M109.008938

54. Ezashi T, Telugu BP, Alexenko AP, Sachdev S, Sinha S, Roberts RM. Derivation of induced pluripotent stem cells from pig somatic cells. Proc Natl Acad Sci USA. (2009) 106:10993-8. doi: 10.1073/pnas.0905284106

55. Erharter A, Rizzi S, Mertens J, Edenhofer F. Take the shortcut - direct conversion of somatic cells into induced neural stem cells and their biomedical applications. FEBS Lett. (2019) 593:3353-69. doi: 10.1002/1873-3468.13656

Conflict of Interest: The authors declare that the research was conducted in the absence of any commercial or financial relationships that could be construed as a potential conflict of interest.

Publisher's Note: All claims expressed in this article are solely those of the authors and do not necessarily represent those of their affiliated organizations, or those of the publisher, the editors and the reviewers. Any product that may be evaluated in this article, or claim that may be made by its manufacturer, is not guaranteed or endorsed by the publisher.

Copyright (C) 2022 Chakritbudsabong, Sariya, Jantahiran, Chaisilp, Chaiwattanarungruengpaisan, Rungsiwiwut, Ferreira and Rungarunlert. This is an open-access article distributed under the terms of the Creative Commons Attribution License (CC BY). The use, distribution or reproduction in other forums is permitted, provided the original author(s) and the copyright owner(s) are credited and that the original publication in this journal is cited, in accordance with accepted academic practice. No use, distribution or reproduction is permitted which does not comply with these terms. 